\title{
Commentary
}

\section{Coeliac disease: how much of what is toxic to whom?}

The cause of coeliac disease remained an enigma until the early 1950s when the Dutch paediatrician, W K Dicke made his seminal observation that gluten, the major storage protein of wheat cereal, is essential to the pathogenesis of the disease. ${ }^{1}$ Removal of gluten from the diet permits virtually complete recovery of the intestinal mucosa and reversal of the clinical and laboratory abnormalities. The precise mechanism whereby gluten causes damage to certain susceptible individuals has still to be elucidated. Many features of the disorder are in keeping with central involvement of the immune response, displaying a delayed-type hypersensitivity reaction to gluten. However, other features are in keeping with a so called autoimmune disease: these include a notable association with MHC class II genes (in particular HLA-DQ2), a female preponderance of approximately 3 to 1 , and the presence of an autoantibody, the anti-endomysial antibody. Thus, coeliac disease could represent an unique example of autoimmune disease, where the disease process is absolutely dependent on continued exposure to an exogenous, dietary protein.

In 1983, Chorzelski and colleagues reported the association between gluten sensitive disease and circulating IgA anti-endomysial antibodies. ${ }^{2}$ In 1997, the nature of the endomysial antigen was discovered to be tissue transglutaminase $\mathrm{e}^{3}$ and this gave an intriguing insight into the link between gluten and anti-endomysial antibodies: gliadin, a subfraction of gluten, has high substrate affinity for this transglutaminase enzyme. ${ }^{4}$ Thus, through mechanisms yet to be clarified, the presence of gluten drives an autoimmune response to transglutaminase. A deluge of clinical studies followed, all confirming the absolute specificity of anti-endomysial antibodies for gluten sensitive disease. A similar finding was made in our centre but the antibody test was less sensitive, with $84 \%$ of untreated patients antibody positive. ${ }^{5}$ Remarkably, the presence of anti-endomysial antibody is specifically associated with continuing exposure to gluten and only indirectly with the intestinal inflammatory process. Thus, patients may have only very subtle evidence of a pathological lesion, such as raised intraepithelial lymphocytes, and yet be antiendomysial antibody positive. Furthermore, following a gluten-free diet, anti-endomysial antibodies may disappear despite the presence of continuing intestinal inflammation.

However, making the diagnosis is only the fist step in treating the patient with coeliac disease. Compliance with therapy - that is, a strict gluten-free diet, may be a more formidable task. Treatment of the more obvious symptomatic manifestations of coeliac disease is the initial goal of patients and most are also aware of the increased risk of malignancy if gluten remains in the diet. Furthermore, it is also known that an incomplete gluten-free diet may result in chronic malabsorbtion with complications such as osteoporosis. ${ }^{6}$ None the less, poor compliance with a strict gluten-free diet is common and up to $50 \%$ of patients "break" their diet on a regular basis. Reasons for this vary. Some patients receive inadequate instruction and supervision of what the diet entails. We believe that attendance at a designated Coeliac Clinic with regular clinical, laboratory and dietetic review helps the patients to achieve their goal. Of course, some patients may adopt a laissez faire approach and so long as they remain relatively asymptomatic, they are prepared to eat some gluten containing foods. This approach is particularly prevalent in younger patients, especially during the teenage years.

Factors which impact on compliance include the relative limitations of the diet, inadequate labelling of foods, uncertainty with respect to meals prepared outside the home, increasing use of gluten as an additive to food and pharmaceutical products, and uncertainty as to what is required for the diet to be absolutely gluten-free. To help tackle the problem of dietary monotony, studies were performed to investigate whether oats could be safely added to the coeliac diet. The practice has been to exclude oats, on the basis of its possible toxicity. However, in three recent studies, no disease activation was evident in patients eating oats. ${ }^{7-9}$ Despite these reports, reluctance to add oats to the gluten-free diet remains.

The debate as to whether oats is toxic leads immediately to the question, precisely what component of gluten is toxic. It is generally accepted that the major, damage inducing component is contained in the alcohol soluble fraction of gluten, termed gliadin. Similar alcohol fractions of rye and barley, two further cereals which belong to the same Triticum genus as wheat, are also considered to contain the toxic components. The alcohol soluble fractions, known as prolamins, of all three cereals display chemical homology and this includes repeated sequences of proline and glutamine. Evidence has accumulated that one toxic component of the gliadin molecule is contained within a 19 amino acid sequence at the $\mathrm{N}$-terminal end of the molecule. ${ }^{10}$ Based on evidence of other gliadin toxic sequences and sequences found in barley and rye, it has been proposed that the sequence QQQPFP may be essential for toxicity. It is interesting to observe that oats contains a very similar sequence, QQQPFV but it should be noted that valine replaces the final proline in the gliadin sequence. This information may be very informative concerning identification of toxic cereal sequences and is in keeping with the notion that the properties of valine are chemically unfavourable for a toxic effect. This could explain data suggesting that oats can be safely included in the gluten-free diet.

Central to the issue of dietary compliance, consideration has been given to methods of ensuring that commercially prepared foods are safe for coeliac patients. According to proposed new regulations, $10 \mathrm{mg}$ gliadin per $100 \mathrm{~g}$ food product will be the maximum allowed in food deemed to be gluten-free. Various assay systems including reversephase HPLC, polymerase chain reaction and mass spectrometry are used to test foods for the presence of gluten. Other tests are based on the detection of antigens in food and in this issue (see page 190) a highly sensitive capture ELISA assay is reported. In this ELISA system, gliadin is detected by a monoclonal antibody raised against the 19 mer gliadin peptide described earlier and the authors 
believe that the reactive epitope may include the QQQPFP sequence. This test may prove useful in determining which foods are gluten-free. None the less, it should be pointed out that oats flour cross-reacted in this assay system and this could give rise to a spurious result - that is, suggesting a food was not safe, even though oats was the only cereal present. This raises the old conundrum, can the antigenicity of a cereal be simply equated with its toxicity for coeliac patients? To establish peptide sequence toxicity, more fundamental studies may be required and these could include investigation of cereal peptide interaction with tissue transglutaminase.

C F FEIGHERY

Department of Immunology, St fames's Hospital,

fames's Street,

Dublin 8,

Ireland
1 Dicke WK. Coeliakie. PhD thesis. Utreacht, The Netherlands: Univ of Utreacht, 1950.

2 Chorzelski TP, Sulej T, Tchorzenwska H, et al. IgA class endomysium antibodies in dermatitis herpetiformis and coeliac disease. Ann NY Acad Sci 1983;420:325-34.

3 Dieterich W, Ehnis T, Bauer M, et al. Identification of tissue transglutaminase as the autoantigen of celiac disease. Nat Med 1997;3:797-801.

4 Bruce SE, Bjarnason I, Peters TJ. Human jejunal transglutaminase: demonstration of activity, enzyme kinetics and substrate specificity with special relator to gliadin and coeliac disease. Clin Sci 1985;68:573-9.

5 Feighery C, Weir DG, Whelan A, et al. Diagnosis of gluten-sensitive enteropathy: is exclusive reliance on histology appropriate? Eur f Gastroenenteropathy: is exclusive reliance
terol Hepal 1998 (in press).

6 Keaveny AP, Freaney R, McKenna MJ, et al. Bone remodelling indices and secondary hyperparathyroidism in celiac disease. Am F Gastroenterol 1996; 91:1226-30.

7 Janthuinen E, Pikkarainen P, Kemppainen T, et al. A comparison of diets with and without oats in adults with celiac disease. N Eng f Med 1995;333: 1033-7.

8 Srinivasan U, Leonard N, Jones E, et al. Absence of oats toxicity in adult coeliac disease. BMF 1996;313:1300-1.

9 Hardman CM, Garioch JJ, Leonard JN, et al. Absence of toxicity of oats in patients with dermatitis herpetiformis. N Engl f Med 1997;337:1884-7.

10 Sturgess P, Day P, Ellis H, et al. Wheat peptide challenge in coeliac disease. Lancet 1994;343:758-61. 